\title{
Departamento de Medicina Social A reestruturação e os novos tempos
}

\author{
Juan S. Yazlle Rocha \\ Docente. Departamento de Medicina Social. Faculdade de Medicina de Ribeirão Preto - USP
}

\section{A Faculdade de Medicina de Ribeirão \\ 1 Preto da Universidade de São Paulo} existência completados em 2.002; esta já é uma razão suficiente para refletir sobre os caminhos andados, as realizações, as opções assumidas e as oportunidades não consideradas. Há 10 anos atrás, em comemoração aos 40 anos da FMRP tal tarefa foi realizada, em parte, com o lançamento de um número especial da revista Medicina, na qual foram publicados trabalhos de análise e avaliação da trajetória seguida por todos os departamentos da instituição ${ }^{(1)}$. Pode-se pensar que pouco haveria a acrescentar ao que foi dito e assinalado há uma década. Todavia, os 50 anos de uma instituição pública marcam, no mínimo, a renovação total do quadro docente e de servidores fundadores, facilitando a passagem a novas idéias, que podem inspirar novos projetos, por diferentes caminhos.

Se isso não fosse suficiente, tivemos, nos últimos 10 anos, um período de grandes e significativas mudanças. A década de 90 trouxe, no plano internacional, a queda dos regimes socialistas na Europa Oriental, com o consequiente predomínio da influência norte-americana no mundo. Foi a década da conscientização do fenômeno da globalização (iniciada, nos países centrais, na década anterior) com fortes transformações na Economia, a imensa expansão da informatização e a revolução nos sistemas de acumulação e difusão do conhecimento, maximizada pela internet, a queda de muitas tradições, e a reafirmação de outras, étnicas e religiosas, principalmente ${ }^{(2)}$. No cenário nacional, a década coincide com a retomada da democratização, a vigência da nova Constituição Federal (1.988), que propiciou a Reforma do Estado e da Administração Pública (1.995), a realização das Refor- mas da Saúde e da Educação - implantação do Sistema Único de Saúde (a Lei 8.080 é de 19/09/90), a Lei de Diretrizes e Bases da Educação (a Lei 9.394 é de 20/12/96).

As reformas trouxeram, em seu bojo, a transformação do papel do Estado e mudanças nas suas relações com a Sociedade, o que veio promover significativas alterações de caráter setorial. O Estado, agora como poder regulador mais do que interventor, na Economia, alterou significativamente a situação dos setores sociais, mormente diante dos problemas do ajuste fiscal; aceitou-se que a saúde é um componente essencial do desenvolvimento humano e que este requer, além da saúde e da educação, crescimento econômico, ambiente sadio e liberdades humanas. Firmou-se o conceito ampliado do que é público, acrescentando-se ao setor público estatal as entidades públicas não estatais, abrindo-se o caminho para se tornarem publicas as atividades que antes eram responsabilidade exclusiva do Estado.

Especificamente, no campo da Saúde Pública, no Continente Americano, gerou-se uma crise de identidade, que motivou sucessivos seminários acerca da crise da saúde pública tradicional ${ }^{(3)}$, a necessidade de repensar a prática ${ }^{(4)}$, e que terminaria com a redefinição das funções essenciais da Saúde Públi$\mathrm{ca}^{(5)}$. A Saúde Pública era entendida por muitos "como parte intrínseca do Estado, onde este aparece como a única garantia para poder superar os interesses setoriais e/ou individuais e favorecer a possibilidade de ações dirigidas a conjuntos sociais, tanto em termos de cura como de prevenção"(6).

Atualmente, as práticas sociais da Saúde Pública são entendidas como responsabilidade do Estado, com atribuições específicas dos Ministérios de 
Saúde e, também, de órgãos públicos, não estatais, a cargo de instituições da sociedade civil $^{(7)}$, que são:

1) construção de ambientes saudáveis e melhoria das condições de vida;

2) desenvolvimento e fortalecimento de uma cultura da vida e da saúde;

3) geração de inteligência em saúde;

4) assistência às necessidades e demandas em saúde;

5) garantia de seguridade e qualidade de bens e serviços de saúde;

6) intervenção sobre riscos e danos coletivos à saúde.

No Brasil, a implementação do Sistema Único

de Saúde, ainda que com avanços, recuos e atrasos, delineou uma tendência constante: o fortalecimento do sistema público, sua descentralização, a criação dos sistemas municipais e estaduais de saúde, a participação da comunidade em conselhos e conferências, nos três níveis de governo, as transferências de recursos financeiros fundo a fundo, a transformação do modelo assistencial existente, centrado sobretudo nas ações curativas, através da implementação do Programa de Saúde da Família e, mais recentemente, a aprovação de uma Emenda Constitucional (PEC 029) capaz de garantir um recurso financeiro mínimo às ações do SUS.

O desafio de implementar tais diretrizes passa pela necessária capacitação de recursos humanos e desenvolvimento de pesquisas que orientem a gestão do sistema e as decisões políticas da área. Como seria esperado, esse conjunto de fatores ocasionou profundas mudanças na Educação Médica - no curriculum médico, nos campos de atuação e nas práticas docentes e discentes.

\section{AS MUDANÇAS NA FMRP-USP}

A transformação do Sistema Público de Saúde, com a definição de um modelo nacional, fundamentado na descentralização das ações para Estados e Municípios, na regionalização e hierarquização da rede assistencial, na transferência às municipalidades da atribuição da gestão da saúde, a ênfase na assistência primária à saúde, entre outras mudanças, veio provocar ajustes nos Programas de Educação Médica.

O Hospital das Clínicas, que atendia, exclusivamente, pessoas sem cobertura de qualquer sistema social de assistência médica - chamados de "indigentes"- diante da universalização da assistência previdenciária, pelo SUDS, primeiro, e pelo SUS, depois, teve que rever aquela medida e aceitar atender a pacientes previdenciários, mediante convênio específico (provavelmente o HC-FMRP foi um dos últimos hospitais-escola do país a aderir ao atendimento a pacientes previdenciários, em 1.988). Mantido até então, exclusivamente, com recursos do Tesouro do Estado de São Paulo, o HC passou a contar, a partir desse convênio, com o aporte de novos recursos, provenientes dos repasses do INAMPS, primeiro, e do Ministério da Saúde, depois. Junto, veio a questão da administração e aplicação desses recursos; diante da rigidez do modelo de administração pública burocrática mesmo sendo o HC uma autarquia, optou-se, ainda em 1.988, por criar uma Fundação de Apoio ao Ensino, Pesquisa e Assistência (FAEPA) que flexibiliza a aplicação dos recursos e permite linhas de financiamentos para atividades de pesquisa e de extensão dos docentes e dos departamentos da unidade. A cultura institucional do Hospital das Clínicas viria adaptar-se a essas profundas mudanças, de forma gradual, num processo ainda em andamento.

A Faculdade de Medicina de Ribeirão Preto iniciou, em 1.993, a Reforma Curricular, com a condensação dos conteúdos teóricos dos ciclos Básico e Pré Clínico, em 2 anos de duração cada um, eliminando repetições e priorizando conteúdos, e transferindo algumas práticas para o Internato, agora ampliado para 2 anos. Foram introduzidas precocemente, práticas de saúde no Curso Médico, na Disciplina de Iniciação à Saúde, oferecida aos alunos do primeiro ano, levando a participação discente a ambulatórios da rede básica de serviços de saúde, a visitas domiciliares e a estabelecimentos comunitários, como creches e asilos. Em 1997, o primeiro ano do Internato do novo curriculum estava centrado na atenção primária à saúde, com práticas discentes sobretudo na rede básica de serviços de saúde, em instituições comunitárias e atuação com a própria comunidade, nas oito semanas de duração do Estágio Integrado e Medicina Comunitária I, além da Medicina Comunitária II, com 4 semanas, mantida no segundo ano de Internato.

A implantação e desenvolvimento do SUS transformou definitivamente o hospital-escola em instituição de nível terciário, pólo regional de assistência, adequado à pesquisa e treinamento de recursos humanos especializados e super especializados. Assim, a formação médica passou a requerer espaços institucionais "extra-muros", de forma ampla e crescente. O Centro de Saúde-Escola, criado e mantido pelo Departamento de Medicina Social (DMS) desde 1.979 foi absorvido pela Faculdade de Medicina em 1.998 - embora o Diretor Técnico do mesmo seja escolhido pelo Diretor da FMRP, de lista tríplice, elaborada pelo 
Conselho do Departamento. No mesmo ano, foi aprovado o Regimento do Centro de Saúde-Escola como órgão subordinado à Diretoria da FMRP.

No ano seguinte, 1.999, o DMS deu início ao primeiro Programa de Residência Médica em Medicina Geral e Comunitária, com enfase em Saúde da Família, no Estado de São Paulo; embora interdepartamental, o programa está fortemente alicerçado na participação de docentes do DMS; nesse mesmo ano, 1.999, foi criado e aprovado o Regimento do Pólo Norte/Oeste Paulista de Formação Acadêmica e Capacitação de Recursos Humanos para a Saúde da Família. As mudanças implementadas na Faculdade de Medicina de Ribeirão Preto são coerentes com as diretrizes nacionais na área da saúde, propiciando aos seus alunos, de um lado, um ambiente educacional integrado nas transformações do setor, e, de outro, colaborando com o desenvolvimento e consolidação do Sistema Único de Saúde e, mais especificamente, na estruturação da rede de serviços de saúde, desde a atenção primária e saúde da família (nível primário) até o hospital universitário (nível terciário).

\section{BREVE HISTÓRICO E ANTECEDENTES DO DMS}

Ao criar, em 1.954, de forma pioneira, o primeiro Departamento de Higiene e Medicina Preventiva numa Escola Médica, no Brasil, a Faculdade de Medicina de Ribeirão Preto procurou inovar no modelo de ensino médico: integrou os conteúdos tradicionais da Higiene ao que havia de mais avançado naquele tempo: a Medicina Preventiva; esta, preconizando a visão holística do paciente, sua família e a comunidade, acrescentou a Epidemiologia e a Bioestatística aos referenciais da higiene e saúde pública no estudo do fenômeno biológico e social da saúde e da doença, tornando-se referência na Educação Médica LatinoAmericana. O departamento desenvolveu um programa de visitas domiciliares a casos escolhidos entre os pacientes atendidos no ambulatório de Medicina Preventiva e foi pioneiro ao criar o Instituto de Medicina Rural, no município de Cássia dos Coqueiros, em 1.964; participou da criação e implantação de um serviço de Medicina Comunitária em área urbana, o Centro Médico Social Comunitário de Vila Lobato, junto com os Departamentos de Pediatria e de Obstetrícia e Ginecologia, 1.970 .

O Departamento de Higiene e Medicina Preventiva foi pioneiro, também, na abertura da Residên- cia Médica em Medicina Preventiva, a partir de 1.966, e no Mestrado e Doutorado em Medicina Preventiva, a partir de 1971, já com o nome atual de Departamento de Medicina Social. Em fins da década de 70 e início da de 80, o DMS passou a incorporar o pensamento crítico das ciências humanas, criando as disciplinas de Ciências Sociais Aplicadas e Planejamento e Administração em Saúde, acompanhando o movimento desencadeado no âmbito da Saúde Pública e Medicina Preventiva, alinhando-se à corrente do setor que se identifica como Medicina Social, fazendo jus ao novo nome.

O período que compreende a década de 70 até a metade da de 80 é o tempo da gestação e desenvolvimento do Movimento da Reforma Sanitária, com aprofundamento da crítica teórica e conceitual da Saúde Pública e da Medicina Preventiva e dos modelos teóricos e práticos dali derivados: a Medicina Comunitária e a Integração Docente Assistencial. Ao absorver conteúdos das ciências humanas, principalmente da Sociologia e da Política, desenvolveu-se um pensamento crítico, inicialmente calcado nas idéias de reformadores europeus, como Rudolf Virchow, Jules Guérin e William Farr; amparado nas leituras de Marx e Gramsci, entre outros, o movimento criou seus teóricos latino-americanos: Juan César García, Cecília Donnangelo, Cristina Laurell, Antônio Sergio Arouca, Jaime Breilh, Edmundo Granda, Guilherme Rodrigues da Silva e muitos outros. Tudo isso representou o nascimento de uma Escola Latino-Americana de pensamento na saúde, que elaborou uma nova compreensão das relações entre a saúde e a sociedade, entre trabalho e doença, entre classes sociais e Medicina, entre conhecimento e produção industrial na saúde, entre política e democracia, poder e controle social, etc., e que veio a constituir a Saúde Coletiva, no Brasil.

Esse saber militante gerou sua própria práxis, o Movimento da Reforma Sanitária Brasileira, que viria a constituir-se em pensamento hegemônico na $8 \mathrm{a}$. Conferência Nacional de Saúde, em Brasília (1.986), sentando as bases para a criação do Sistema Único de Saúde (SUS).

\section{O DMS NA DECADA DE NOVENTA}

Tendo em vista a necessidade urgente de implementar a Reforma Sanitária e a crescente importância da área, em 1.991, o DMS propôs e aprovou a criação de Cursos de Especialização em Saúde 
Pública, Epidemiologia, Planejamento e Administração em Saúde e Saúde do Trabalhador; todavia, para implementar essa proposta do DMS seria necessária a ampliação do quadro docente, o que não encontrou respaldo na Direção da FMRP e nem na Reitoria da USP, naquela época. Mesmo assim, o DMS ofereceu 4 Cursos de Especialização em Saúde Pública a profissionais da cidade e região. Houve grande demanda de profissionais qualificados para assumirem postos de direção nos serviços de saúde locais, regionais, estaduais e federais - sendo que o DMS, através dos seus docentes, deu sua colaboração prática na construção do SUS. Aquele foi, provavelmente, o momento de auge do modelo preventivista e de Medicina Social do DMS.

No início da década de 90, o DMS chegou a contar com 14 docentes, quando outros departamentos similares, no Estado de São Paulo possuem sempre mais de 20 docentes na área. Impossibilitado de expandir-se, o DMS começou a sofrer o desgaste de administrar os importantes serviços de extensão, que deram suporte a pesquisas e à formação de recursos humanos próprios e de outras áreas e unidades do Campus: o Centro de Saúde-Escola, o Centro Médico Social Comunitário Pedreira de Freitas, o Centro de Processamento de Dados Hospitalares e o Núcleo de Vigilância Epidemiológica. Entretanto, a falta de perspectivas de crescimento levou ao afastamento de dois docentes que, mais tarde, pediram demissão da USP; um outro docente passou a prestar serviços à Secretaria de Estado da Saúde e, posteriormente, pediu aposentadoria, e o quadro ficou reduzido a 11 docentes.

Os esforços para a reposição do quadro foram infrutíferos, resultando no prejuízo das atividades acadêmicas: a avaliação, pela CAPES, dos Programas de Mestrado e Doutorado para 1.994/1.995 foi B e C, respectivamente. A previsão da crise e os alertas dirigidos às autoridades acadêmicas, relatando a iminência de aposentadorias de vários docentes, foram desconsiderados totalmente, bem como os efeitos que isso produziria nas atividades do departamento.

Em 1.997, sobreveio a crise anunciada: ocorreu a aposentadoria de 4 docentes - e a morte de um quinto - reduzindo o quadro a 6 professores, o que inviabilizou totalmente os Programas de Mestrado e Doutorado e a Residência Médica, que tiveram que ser suspensos, ameaçando, ainda, a continuidade das áreas e programas do departamento.

\section{O PROJETO DE REESTRUTURAÇÃO}

Em fins de 1.997, as autoridades acadêmicas reconhecem a crise instalada e, sendo a Medicina Social uma das áreas básicas na educação médica, foi decidida a revitalização do departamento. Todavia, era evidente a necessidade de "atualizar" a inserção do DMS na Faculdade de Medicina, levantando a questão da renovação do projeto departamental, para atender a algumas demandas internas, como desenvolvimento de cursos e assessorias em métodos quantitativos de investigação, epidemiologia clínica e a coordenação e supervisão de atividades discentes em atenção primária. No mesmo sentido, no Departamento de Medicina Social já havia a forte impressão de uma situação de crise ou esgotamento do tipo de proposta existente, pela constatação da queda da demanda ao programa de residência médica, voltado à formação de sanitaristas, decorrente da contração do mercado de trabalho e pelas dificuldades de serem mantidos os programas de Mestrado e Doutorado, dirigidos exclusivamente a médicos, em decorrência da estruturação multiprofissional da área da saúde coletiva. Finalmente, a decisão política do Ministério da Saúde de mudar o modelo assistencial brasileiro, promovendo as ações de atenção primária e de saúde da família, consolidou nossa convicção de que era necessário mudar o projeto departamental, e criou a necessidade de contar com programas destinados à formação dos recursos humanos adequados - tanto para o setor de serviços, com vagas asseguradas no mercado de trabalho, praticamente em todo o país, como para a docência que levassem à inserção e desenvolvimento dessas práticas nas escolas médicas. Foi nesse cenário que o grupo remanescente de docentes do DMS definiu as diretrizes estratégicas da reestruturação do Departamento de Medicina Social.

A organização e a estruturação das atividades discentes, no primeiro ano do Internato, desenvolvidas em atenção primária, originaram o Estágio Integrado, que demandou a criação do Centro de Atenção Primária e de Saúde da Família, 1.997. O referido centro, interdepartamental congregando docentes dos departamentos de Medicina Social, Pediatria, Obstetrícia e Ginecologia e Cirurgia recebeu a atribuição de propor a organização e o desenvolvimento das atividades de pós-graduação, lato sensu e stricto sensu, na área.

Além do apoio da direção da Faculdade de Medicina (Diretor e Vice-Diretor), foi importante con- 
tar com o apoio da Comissão de Graduação que, pela subcomissão do Internato, interveio a favor da concessão de 2 claros docentes, em caráter emergencial, para dar suporte às atividades discentes de atenção primária e saúde da família. Duas outras vagas foram conseguidas, como reposição do colega falecido e de um dos aposentados. É preciso lembrar que, por razões orçamentárias, a Universidade de São Paulo tinha suspendido totalmente as reposições de docentes em casos de aposentadorias; também estavam suspensas as autorizações de contratação em unidades que não tivessem implementado a reestruturação departamental, como era o caso da FMRP. Um quinto docente viria, pela abertura de concurso, para prover um cargo de professor titular, decorrente de uma das aposentadorias, tendo sido possível interessar um experiente professor de Medicina Preventiva da UNIFESP a concorrer ao mesmo e a transferir-se para Ribeirão Preto.

Com a definição dessas perspectivas, o Conselho do Departamento aprovou, em abril de 1.998, com a participação de todos os docentes, as seguintes diretrizes: priorizar a área da atenção primária e saúde da família, revitalizar o setor de métodos quantitativos e reivindicar o retorno do setor de Bioestatística ao $\mathrm{DMS}^{(8)}$. Essas diretrizes aparecem nas Metas do Plano Departamental, qüinquienal, aprovado pelo Conselho do Departamento: capacitar o DMS a coordenar e participar da organização e desenvolvimento de Programas e Cursos em Atenção Primária e Saúde da Família, na graduação e na pós-graduação; formar recursos humanos, no Mestrado, para trabalhar na gestão de distritos sanitários do Sistema Único de Saúde; intensificar a pesquisa original e publicações em revistas de circulação internacional; formar docentes pesquisadores em Epidemiologia e Atenção à Saúde, no Doutorado; manter e desenvolver as atividades de extensão - centros e núcleos essenciais para o ensino e pesquisa na área.

No mesmo documento, aprovou "transformar a residência em Medicina Preventiva em Programa de Atenção Primária e Saúde da Família para médicos e enfermeiros" - o qual teria início em 1.999. No Mestrado, aprovou substituir o Programa de Medicina Preventiva por um Curso de Saúde da Comunidade, com o objetivo de formar docentes, pesquisadores e dirigentes de serviços, aptos a investigar o processo saúde e doença, a organização dos serviços de saúde e os modelos de assistência, bem como a desenvolver o domínio de métodos e técnicas utilizadas em pesquisas na comunidade; multiprofissional, o curso é dirigido a profissionais como médicos, enfermeiros, dentistas, farmacêuticos, fisioterapeutas, nutricionistas, psicólogos e outros, tendo tido início em 1.999. Decidiuse que, no Doutorado, o Programa de Medicina Preventiva enfatizaria a formação de docentes pesquisadores em seus 2 setores mais tradicionais: Epidemiologia e Atenção à Saúde - projeto ainda não implementado. Decidiu-se, também, nos Cursos de Especialização, a participação da Capacitação em Atenção Primária e Saúde da Família para médicos e enfermeiros.

A opção departamental e a participação dos docentes do departamento nas atividades de atenção primária e saúde da família foram decisivas para a elaboração do projeto que levou à criação do Pólo Norte Oeste de Formação e Capacitação de Recursos Humanos para a Saúde da Família, incluindo a Escola de Enfermagem do Campus da USP de Ribeirão Preto, a Faculdade de Medicina de São José do Rio Preto e a Universidade Federal de São Carlos, abrangendo as regiões de Araçatuba, Araraquara, Barretos, Franca, Ribeirão Preto e São José do Rio Preto.

\section{AVALIAÇÃO E TENDÊNCIAS}

A tendência posterior das políticas de saúde, no país, e dos programas e atividades, na FMRP, veio confirmar o acerto da estratégia de mudança implementada no DMS. Aprovada a reestruturação departamental, na FMRP, em 2.000, e diante da criação de dois novos cursos de graduação - Fisioterapia e Terapia Ocupacional - além do risco de novas aposentadorias, o DMS recebeu autorização para a contratação de mais 3 docentes: um para o Setor de Métodos Quantitativos, outro para o Setor de Planejamento e Gestão em Saúde e, o terceiro, para a disciplina de Ciências Sociais Aplicadas à Saúde. Ganhou, ainda, a concessão de 2 Bolsas-Docente para a área de métodos quantitativos, custeadas por Convênio FMRP \& FAEPA.

$\mathrm{O}$ aparecimento de um espaço dedicado à atenção primária e à saúde da família, como área acadêmica, reclama várias reflexões. A primeira é a constatação que representa a introdução, na Escola Médica, de um objeto novo de pesquisa e trabalho, carente, portanto de elaborações teóricas e práticas; a segunda é que a nova área representa a possibilidade de construção de uma ponte de ligação entre a Medicina 
Curativa e a Saúde Pública, o que significa que o melhor local para essa construção é o interior das escolas médicas, muito embora os primeiros programas de ensino do PSF tenham surgido, no Brasil, em escolas de Saúde Pública; a terceira é que a área representa, também, a possibilidade de formação de profissionais, e de construção de práticas, comprometidos com a saúde integral, isto é, uma única equipe ou programa, voltados à promoção da saúde, prevenção de doenças e ao diagnóstico precoce, tratamento e reabilitação. Entretanto, é necessário destacar que a proposta de assistência à saúde da família, em desenvolvimento no Brasil, difere significativamente dos programas desenvolvidos em outros países. Em grande parte, isso ocorre porque o PSF, no Brasil, foi gestado com forte influência do pensamento crítico da saúde coletiva latino-americana; a incorporação de conceitos, como a determinação social da saúde-doença, o caráter de classes das práticas da saúde, o controle social e a participação da comunidade, entre muitos outros, fazem a diferença. São elementos que impedem que se identifique a atual situação dos Departamentos de Medicina Preventiva e Social como de "um retorno ao passado" - quando se desenvolviam as clínicas de famílias e as visitas domiciliares e tentava-se promover o desenvolvimento das comunidades.

Atualmente, as escolas médicas, no Brasil, não possuem espaços acadêmicos voltados para o objeto da atenção primária e a saúde da família; os programas que desenvolvem tais atividades recebem forte apoio dos Departamentos de Medicina Preventiva e Social, embora eles continuem a manter atribuições específicas. É interessante assinalar que, embora o Conselho do Departamento de Medicina Social tivesse aprovado acolher o Centro de Atenção Primária e Saúde da Família, garantindo autonomia de ação para o mesmo, por opção dos docentes participantes do referido centro, este ficou vinculado administrativamente à Diretoria da FMRP, e os docentes mantiveram seus vínculos departamentais originais. Isso pode indicar que a tendência futura será criar, nas escolas médicas, Departamentos de Atenção Primária e Saúde da Família - à maneira das escolas médicas na Inglaterra.
Todavia, as mudanças acima assinaladas alteraram também o interior do campo da saúde coletiva; o vigor da implantação e desenvolvimento do SUS e a força com que o mesmo afeta a organização dos serviços e das práticas no setor levaram à necessidade de destacar as questões de Planejamento e Gestão em Saúde, equilibrando a importância desta área com aquela tradicionalmente conferida à Epidemiologia. Caberá aos departamentos de Medicina Preventiva e Social promover e desenvolver os fundamentos específicos que sustentam a área, isto é, a Sociologia e a Antropologia Aplicadas, a Epidemiologia, o Planejamento e a Gestão de Serviços.

\section{REFERÊNCIA BIBLIOGRÁFICAS}

1 - YAZLLE ROCHA JS; RUFFINO NETO A \& NOGUEIRA JL. Departamento de Medicina Social: 40 anos da FMRP. Medicina, Ribeirão Preto, 25: 74-84, 1992.

2 - GIDDENS A. Para além da esquerda e da direita. Editora UNESP, São Paulo, 1996

3 - FERREIRA JR La crisis. In: ORGANIZACION PANAMERICANA DE LA SALUD. La crisis de la salud pública. Reflexiones para el debate. OPS, Washington, D.C., p.233-236, 1992. (Publicación Científica, 540)

4 - ORGANIZACION PANAMERICANA DE LA SALUD. Desarrollo de la teoria y práctica de la Salud Pública en la Región de las Américas (1990-1997) - Informe Final. OPS, Washington, D.C., 1997. Mimeo.

5 - ORGANIZACION PANAMERICANA DE LA SALUD. Programa de Desarrollo de Recursos Humanos. S, Desafios para la educación en salud pública: la reforma sectorial y las funciones esenciales de salud pública. OPAS, Washington, D.C. 2000. 156p.

6 - MENÉNDEZ EL. Salud pública: Sector estatal, ciencia aplicada o ideologia de lo posible In: ORGANIZACION PANAMERICANA DE LA SALUD. La Crisis de la salud pública. Reflexiones para el debate. OPS, Washington, D.C., p.103-122, 1992. (Publicación Científica, 540).

7 - ORGANIZACION PANAMERICANA DE LA SALUD. Las funciones esenciales de salud pública. In: ORGANIZACION PANAMERICANA DE LA SALUD. Desafíos para la educación en salud pública - La reforma sectorial y las funciones esenciales de salud pública. OPAS, Washington, D.C., cap. 3, 2000

8 - UNIVERSIDADE DE SÃO PAULO. FACULDADE DE MEDICINADE RIBEIRÃO PRETO. DEPARTAMENTO DE MEDICINASOCIAL. Projeto Departamental 1.999-2.003. Ribeirão Preto, 1998, 5p. Mimeo. 


\begin{tabular}{|c|c|c|}
\hline ANOS & DOCENTE & TITULACÄ̃O * \\
\hline $1954-1966$ & JOSÉ UMA PEDREIRA DE FREITAS & CATEDRÁTICO \\
\hline $1955-1965$ & GERALDO GARCIA DUARTE & PROF. ASSOCIADO \\
\hline $1957-1987$ & NAGIB HADDAD & PROF. TITULAR \\
\hline $1959-1961$ & CARLOS LUIZ CAMPANA & INSTRUTOR \\
\hline $1959-1960$ & MARIA LUCILA MILANESI & INSTRUTOR \\
\hline $1960-1997$ & CLARISSE DULCE GARDONYI CARVALHEIRO & PROF. ASSOCIADO \\
\hline $1961-1973$ & JOSÉ ROMERO TERUEL & PROF. LIVRE DOCENTE \\
\hline $1962-1966$ & EUCLIDES CUSTÓDIO DE LIMA FIUHO & PROF. DOUTOR \\
\hline $1963-1975$ & MANILDO FÁVERO & PROF. LIVRE DOCENTE \\
\hline $1966-1997$ & JARBAS LEITE NOGUEIRA & PROF. ASSOCIADO \\
\hline $1967-1997$ & ANTÔNIO RUFFINO NETO & PROF. TITULAR \\
\hline $1970-$ & JUAN STUARDO YAZШE ROCHA & PROF. TITULAR \\
\hline $1970-$ & UILHO ANTÔNNO GOMES & PROF. TITULAR \\
\hline $1973-1994$ & JOSÉ DA ROCHA CARVALHEIRO & PROF. TITULAR \\
\hline 1973 & AMÁBILE RODRIGUES XAVIER MANCQO & PROF. DOUTOR \\
\hline 1974 & ANTÔNIO RIBEIRO FRANCO & PROF. DOUTOR \\
\hline $1974-1997$ & BRENO JOSÉ GUANAIS SIMỖES & PROF. DOUTOR \\
\hline $1975-1979$ & JOAQUIM EUGÊNIO PAZ & PROF. COLABORADOR \\
\hline $1976-1986$ & JOSÉ CARLOS MEDEIROS PEREIRA & PROF. ASSOCIADO \\
\hline $1977-1979$ & AFONSO DINIS COSTA PASSOS & PROF. ASSISTENTE \\
\hline 1978 & ALDAISA CASSANHO FORSTER & PROF. DOUTOR \\
\hline $1980-1985$ & JOEL DOMINGOS MACHADO & AUXIபAR ENSINO \\
\hline $1985-$ & AF ONSO DINIS COSTA PASSOS & PROF. DOUTOR \\
\hline $1985-1993$ & RICARDO JOSÉ SOARES PONTES & PROF. DOUTOR \\
\hline 1988-1998 & NEIRY PRIMO ALESSI & PROF. DOUTOR \\
\hline 1988-1992 & SANDRA AZEVEDO PINHEIRO & PROF. DOUTOR \\
\hline 1998 & AMAURY LEUS DAL FABERO & PROF. DOUTOR \\
\hline 1998 & ELIZABETHMELONI VIEIRA & PROF. DOUTOR \\
\hline 1998 & MILTONROBERTO LAPREGA & PROF. DOUTOR \\
\hline 1999 & LAÉRCIO JOEL FRANCO & PROF. TITULAR \\
\hline $2001-$ & ANTÔNIO DORIVAL CAMPOS & PROF. ASSOCIADO \\
\hline
\end{tabular}

